\title{
Low Temperature Swelling in Beta-SiC Associated with Point Defect Accumulation
}

\author{
Yutai Katoh, Hirotatsu Kishimoto and Akira Kohyama \\ Institute of Advanced Energy, Kyoto University, Gokasho, Uji 611-0011, Japan
}

\begin{abstract}
An experimental technique to characterize irradiation-induced swelling, or isotropic volume expansion, through a combined utilization of medium-to-high energy accelerators and interferometric surface profilometry, was established. The technique was successfully applied to a characterization of swelling behavior in beta-silicon carbide arising from the accumulation of point defects at relatively low temperatures, i.e., $333-873 \mathrm{~K}$, as a function of fluence level, displacement damage rate and irradiation temperature. Swelling rate and swelling at any given fluence level exhibited a negative dependence on irradiation temperature. The saturated low temperature swelling fell on the lower edge of neutron irradiated swelling data band. The influence of displacement damage rate appeared unremarkable. An additional study on the synergistic effect of atomic displacement damage and helium production revealed an enhancement of low temperature swelling in silicon carbide in the presence of helium.
\end{abstract}

(Received October 31, 2001; Accepted January 17, 2002)

Keywords: irradiation effect, silicon carbide, point defect accumulation, swelling, helium effect

\section{Introduction}

Silicon carbide ( $\mathrm{SiC}$ ) and its composites are attractive materials for nuclear and fusion applications as well as combustion, motor generator, aerospace and transport system applications, due to the inherent superior thermo-physical, -chemical and -mechanical properties, irradiation tolerance and nuclear properties. Recent advancement of SiC-based ceramic composites further promises their potential to structural applications in industrial energy systems. ${ }^{1,2)}$

Nuclear environments are known to cause dimensional instability, thermal and electrical transport property changes, and hardness and fracture toughness changes in silicon carbide due to bombardment of energetic neutrons. ${ }^{2)}$ These irradiation effects are also responsible for potential irradiationinduced mechanical property degradation of SiC-based ceramic composites. Mechanistic understandings of irradiation effects in silicon carbide are so far quite limited because of the complexity in point defect behavior in the ceramic compound. ${ }^{3)}$

The objective of this work is to characterize the swelling, as a consequence of irradiation-produced point defect accumulation, in cubic (beta-) $\mathrm{SiC}$ at relatively low temperatures $(<873 \mathrm{~K})$. The low temperature swelling in $\mathrm{SiC}$ is often called 'point defect swelling' in contrast to 'cavity swelling' which occurs only when vacancies are readily mobile at higher temperatures. ${ }^{4)}$

Accelerated charged particles were utilized for defect production to take advantage of excellent controllability in defect production rate and target temperature. ${ }^{5)}$ A dual-beam irradiation technique was employed to specifically explore the influence of helium production through $(n, \alpha)$ reactions in a fusion environment. In the current work, establishment of an experimental scheme that characterizes the irradiation-induced volume instability was also intended.

\section{Experimental Procedure}

Most of the recently developed high performance SiCbased ceramic composites for fusion and nuclear applications consist of (mostly-) polycrystalline $\mathrm{SiC}$ continuous fibers and matrices. The majority of crystallites both in those fibers and matrices are in cubic beta-phase. In this study, therefore, a high purity ( $>99.9995 \%$ ) polycrystalline beta-SiC, with a mass density of $3210 \mathrm{~kg} / \mathrm{m}^{3}$ (corresponds to $100 \%$ density), produced through chemical vapor deposition (CVD) process (Rohm and Haas Co., Woburn, USA) was used as the samples to be irradiated.

The ion beam irradiation was carried out at the DuET Facility, Institute of Advanced Energy, Kyoto University. 5) 4.0 $\mathrm{MeV} \mathrm{Ni}^{3+}$ ions and $5.1 \mathrm{MeV} \mathrm{Si}^{2+}$ ions were used to introduce displacement damage as a 'single-beam' experiment, while an additional beam of energy-degraded $1.0 \mathrm{MeV} \mathrm{He}^{+}$ ions were simultaneously implanted as a 'dual-beam' experiment for the helium effects study. Rates of displacement damage production and helium implantation as a function of distance from the irradiated surface was calculated using SRIM98 code, assuming $35 \mathrm{eV}$ of sublattice-averaged displacement threshold energy. ${ }^{6,7)}$ The damage level was up to $3 \mathrm{dpa}$ (displacement per atom) in majority of the samples and irradiation temperature was up to $873 \mathrm{~K}$. The displacement damage rate was varied within a range of $1 \times 10^{-5}$ to $1 \times 10^{-3} \mathrm{dpa} / \mathrm{s}$. The helium/dpa ratio in the dual-beam irradiation experiment was $60 \mathrm{appm} / \mathrm{dpa}$, which corresponds to the typical ratio of helium production to atomic displacement in the SiC-based first wall of fusion blanket structures. The specimen temperature during irradiation was maintained within $\pm 10 \mathrm{~K}$ of the nominal irradiation temperature at all time.

The dimensional changes were characterized by means of surface profilometry following irradiation through molybdenum grid meshes with $254 \mu \mathrm{m}$ grid spacing. The surface height change as a consequence of irradiation-induced swelling was measured with Micromap ${ }^{\mathrm{TM}}$ interferometric optical surface profiling system (Micromap Inc., Tucson, USA). 

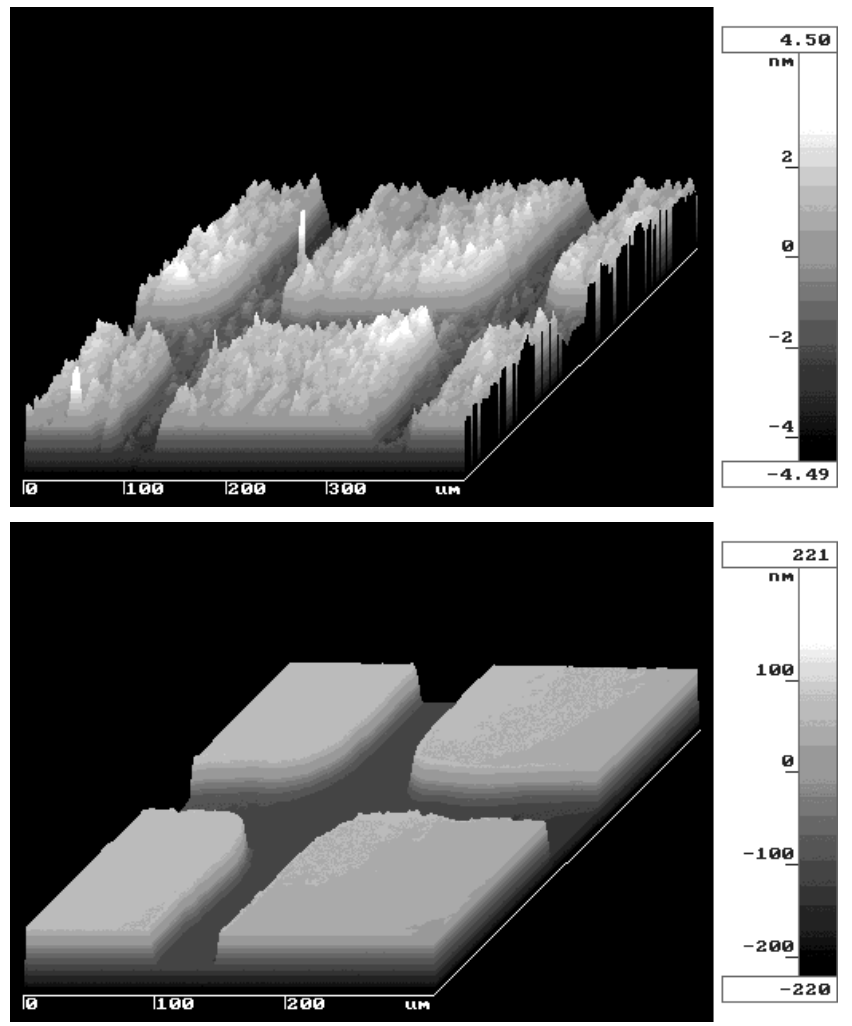

Fig. 1 Surface oblique plots of beta-SiC irradiated with $4 \mathrm{MeV}$ nickel ions at $333 \mathrm{~K}$ to $0.01 \mathrm{dpa}$ (upper) and $1.0 \mathrm{dpa}$ (lower), respectively, through $0.254 \mathrm{~mm}$-pitch molybdenum grid meshes following a surface finish with diamond paste.

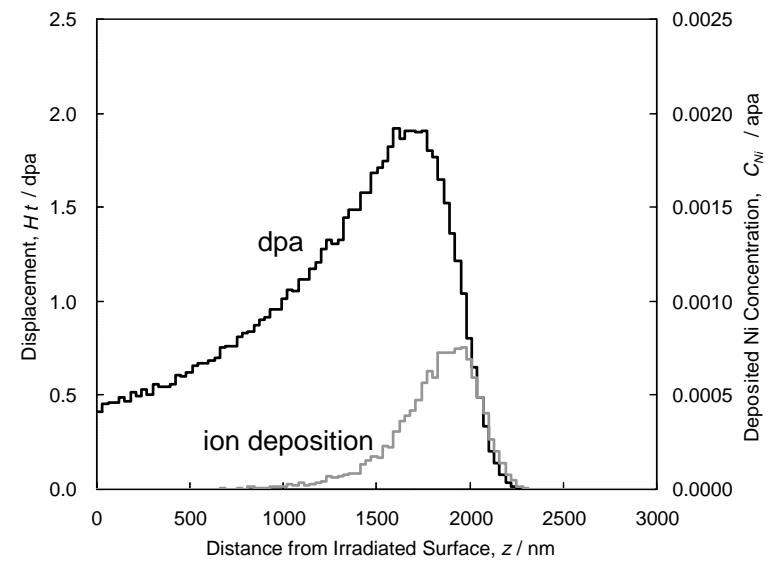

Fig. 2 Profiles of displacement damage and deposited $\mathrm{Ni}$ in irradiated monolithic SiC at a nominal fluence level of 1dpa. Calculation was performed assuming a sublattice-averaged displacement threshold energy of $35 \mathrm{eV}$ and a target mass density of $3.21 \times 10^{3} \mathrm{~kg} / \mathrm{m}^{3}$.

The practical resolution in the profiling is determined by the surface smoothness of the object. Examples of surface profilometry for the cases of 0.01 and $1.0 \mathrm{dpa}$ at $333 \mathrm{~K}$ are presented in Fig. 1 as oblique plots.

Figure 2 shows the calculated depth-profiles of displacement damage (dpa; displacement-per-atom) and stopped ions in silicon carbide for the case of $4 \mathrm{MeV}$ nickel irradiation. A nominal damage level was defined as the dpa averaged over the damage range. The single-beam irradiation-induced swelling $\left(S^{\mathrm{s}}\right)$ was determined as the step-height $\left(\Delta z^{\mathrm{s}}\right)$ divided by the damage range thickness $\left(R_{\mathrm{d}}\right)$;

$$
S^{\mathrm{s}}=\Delta z^{\mathrm{s}} / R_{\mathrm{d}}
$$

According to the calculation in Fig. 2, estimated contribution from ion deposition to the surface height increase is $0.45 \mathrm{~nm} / \mathrm{dpa}$, assuming unchanged atomic density due to the chemical effect of implantation. On the other hand, an estimated sputtering rate of the irradiated surface is $\sim 0.1 \mathrm{~nm} / \mathrm{dpa}$. Since these contributions are sufficiently small compared to the observed swelling-induced height changes, which were more than $20 \mathrm{~nm}$ at $1 \mathrm{dpa}$ in the irradiation conditions in this study, we conclude that the effects of ion deposition and surface sputtering are negligible. However, because of the potential phase instability and atomic density change associated with ion implantation, the influences of ion deposition should carefully by examined in case the irradiation effects to very high fluence levels are of interest.

In the case of dual-beam irradiation, additional swelling due to the co-implantation of helium was calculated by dividing the additional step height change $\left(\Delta z^{\mathrm{d}}-\Delta z^{\mathrm{s}}\right)$ by the thickness of helium implantation range $\left(R_{\mathrm{He}}\right)$, based on the fact that a partial damage range had been co-implanted with helium in the employed experimental set-up. The dual-beam irradiated swelling was derived as a sum of single-beam irradiated swelling and the added swelling due to helium coimplantation;

$$
S^{\mathrm{d}}=\Delta z^{\mathrm{s}} / R_{\mathrm{d}}+\left(\Delta z^{\mathrm{d}}-\Delta z^{\mathrm{s}}\right) / R_{\mathrm{He}}
$$

For a microstructural examination by cross-sectional transmission electron microscopy (XTEM), the irradiated specimens were subjected to a thin-foil processing using a focused ion beam (FIB) micro-processing device. ${ }^{8)}$ The microstructural investigation was performed with JEOL JEM-2010 conventional TEM. Optical microscopy was supplementary utilized for surface feature characterization.

\section{Results and Discussion}

Charged particle bombardment causes a roughening of the incident surface in scales ranging from inter-atomic distance to crystal grain size. For the surface height determination using optical profilometry, roughening in scales of 1 to $10 \mu \mathrm{m}$ most likely imposes a quantitative uncertainty. The root-mean-squared (RMS) surface roughness as a function of displacement damage level at $333 \mathrm{~K}$ is presented in Fig. 3, where the roughness seems to be cumulatively increasing approximately in proportion to the ion fluence. An examination by Nomarski interferometric optical microscopy revealed that the morphology of height variations in the irradiated area was corresponding to crystal grain structure. The degree of the roughness increase was close to or less than $1 \%$ of the swelling in any case. Since it is well known that the irradiation-induced swelling of cubic silicon carbide is isotropic in stress-free conditions, the variation in crystallographic orientation probably caused a relatively minor grainto-grain height variation through interactive constraints in a polycrystalline system in the presence of irradiation-induced plastic flow.

The fluence dependence of swelling was taken within a fluence range of $0.0026-3 \mathrm{dpa}$ at 333 to $873 \mathrm{~K}$ as plotted in 

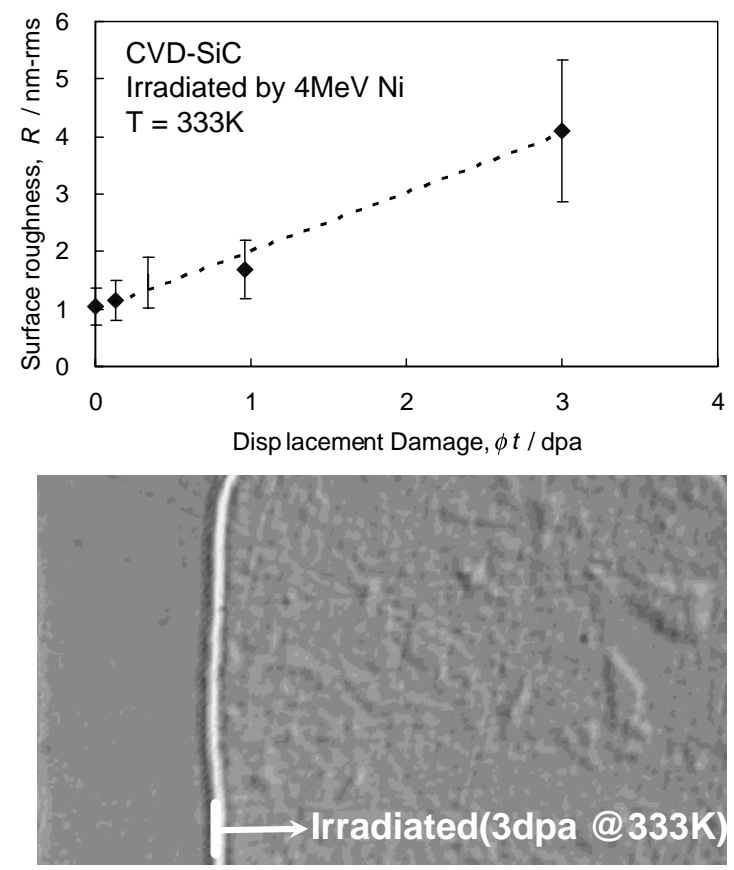

Fig. 3 RMS surface roughness of CVD-SiC as a function of irradiation fluence (upper) and a Nomarski interferometric optical image of the irradiated sample (lower). The dotted line is a least-square linear fit.

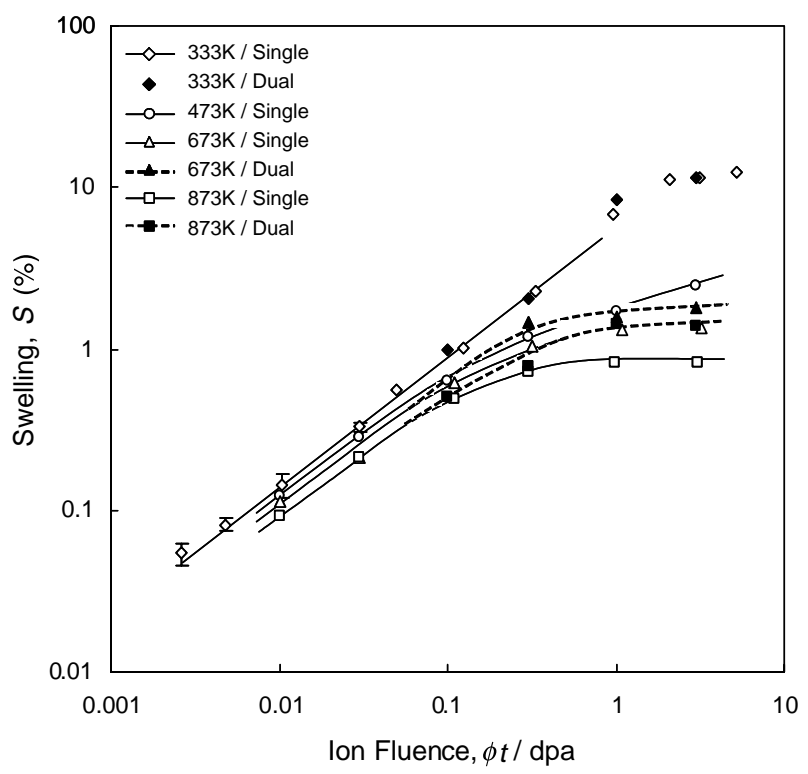

Fig. 4 Fluence-dependence of irradiation-induced swelling at various temperatures.

Fig. 4. The surface roughness was the primary factor that imposed an uncertainty in the swelling measurement. The maximum uncertainty corresponded to $0.02 \%$ of swelling. At $333 \mathrm{~K}$, the swelling increased apparently in proportion to the 0.81 power of the fluence until it approached the saturated value. Physical significance of such dependency may not be able to be clarified, since a very complex defect accumulation and recovery processes, which may incorporate cascade overlapping and/or interactions, Frankel defect recombination, anti-site production, stable cluster production, structural relaxation and dissociation of unstable clusters, etc., are oper- ating in addition to an uneven damage profile in the irradiated volume.

However, if one assumes a linear defect accumulation up to $0.0026 \mathrm{dpa}$ and that each atomic displacement results in a swelling corresponding to one atomic volume for perfect silicon carbide by then, as a very rough estimation, a surviving defect fraction to NRT-dpa can be derived to be $21 \%$. ${ }^{9}$ Since the fluences for apparent cascade overlapping onset in facecentered cubic metals are generally estimated to be within a lower milli-dpa range, ${ }^{10)}$ cascade overlapping should not impose a large error to this estimation with a power law index of 0.81 . The production and recovery behavior of individual defect specie will be studied in more details in separate works.

A rather rapid swelling at near 1 dpa at $333 \mathrm{~K}$ (Fig. 4) indicates a progressive crystalline-to-amorphous phase transition that initiated at the damage peak zone. The swelling due to full amorphization in the single-beam irradiated volume at $5 \mathrm{dpa}$ at $333 \mathrm{~K}$ appeared to be $12.6 \%$. This number falls in a typical range of swelling of originally full-dense silicon carbide associated with neutron-induced amorphization, which is reported to be $11-15 \% .^{11)}$

At temperatures up to $873 \mathrm{~K}$, the apparent defect accumulation rate decreased with the increasing temperature, as shown in Fig. 4. The swelling exhibited a tendency toward saturation at these temperatures. The estimated saturation fluence was smaller at a higher temperature. Hereafter, the swelling at $3 \mathrm{dpa}$ is regarded as a saturated swelling, since a significant swelling increase was not observed after $1 \mathrm{dpa}$ at temperatures $>673 \mathrm{~K}$.

The influence of displacement damage rate on swelling behavior was not very significant but detectable in a consistent way as presented in Fig. 5. At temperature of $333 \mathrm{~K}$, the reduction in displacement rate by one order decreased swelling by approximately $20 \%$ at a given fluence level, however, did not significantly change the swelling exponent. This indicates that thermally activated migration of self interstitial atoms is very likely even at temperature where irradiation-induced amorphization is allowed. The effect of displacement rate within the power-law swelling regime at higher temperatures appeared similar to that at $333 \mathrm{~K}$. However, swelling after deviation from the power-law regime exhibits a reduced sensitivity to displacement rate until it becomes nearly independent of displacement rate at over about $0.3 \mathrm{dpa}$ at both $473 \mathrm{~K}$ and $873 \mathrm{~K}$.

Because vacancy migration is almost prohibited in this temperature range, ${ }^{12)}$ a volumetric swelling should be close to vacancy concentration and, on the other hand, a saturated vacancy concentration is determined by an equilibrium of vacancy production and Frenkel defect recombination through migration of self interstitial atoms (SIA). Therefore, the experimental observation of saturated swelling that is independent of displacement rate suggests that the equilibrium SIA concentration in a saturated swelling regime is proportional to the displacement rate. Such a situation is enabled only when the Frenkel defect recombination rate is much higher than the SIA annihilation rate at other sinks and the sink conditions are not strongly affected by the displacement rate.

In Fig. 6, the swelling at 3dpa obtained in this work is plotted as a function of irradiation temperature, along with reported neutron irradiation data. ${ }^{4)}$ All the ion irradiation data 


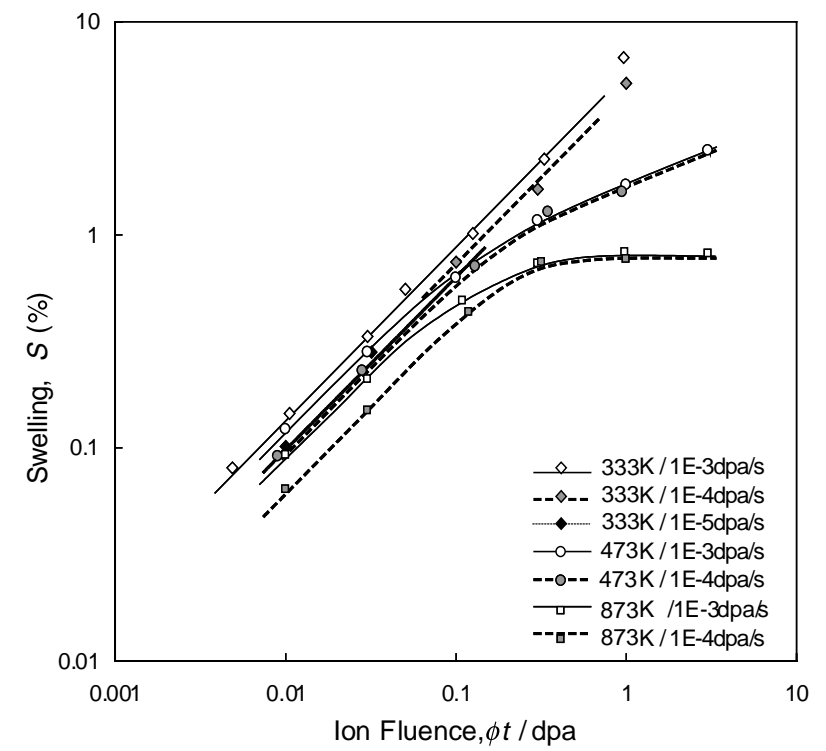

Fig. 5 The influence of displacement damage rate on fluence-dependent swelling behavior of beta-SiC.

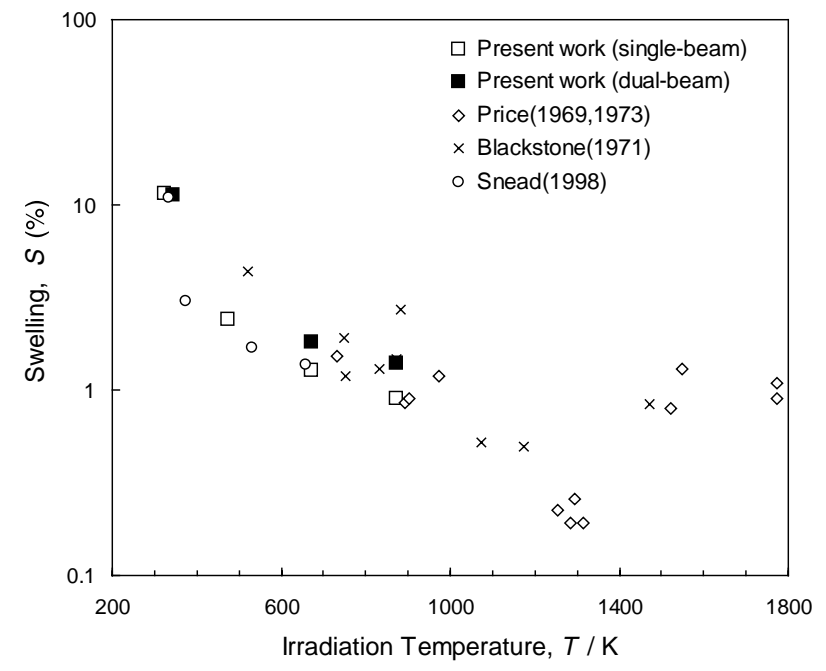

Fig. 6 Temperature-dependence of saturated swelling. Neutron data are from compilation in Ref. 11).

points fall close to the bottom of a neutron data band. Considering the outstanding controllability of irradiation conditions in the present ion irradiation experiment, we suspect that majority of neutron data have been affected by irradiation temperature uncertainty and/or unintentional transient low temperature neutron exposure that is peculiar to the irradiation in fission reactor cores. ${ }^{13)}$ In addition, some of the neutron data might have been affected by transmutation of boron, that had been added as a sintering aid to produce dense materials.

An XTEM image, taken at a $\{111\}$ reflection in a dark-field condition, of the specimen irradiated to $1 \mathrm{dpa}$ at $333 \mathrm{~K}$ is provided in Fig. 7. An amorphous region was identified at around the damage peak depth. An amorphization threshold dose can accurately be determined from the XTEM by overlaying a calculated damage profile. The threshold dose of 1.07 dpa was obtained from this examination in the irradiation condition of $333 \mathrm{~K}$ and $1 \times 10^{-3} \mathrm{dpa} / \mathrm{s}$. The estimated accuracy of threshold dose determination in this procedure is within a few per- cent, although the conditions for calculation by SRIM may exert a more significant uncertainty. This data appeared to fall strictly on the reported line of temperature dependence of amorphization threshold dose for cases both hexagonal and cubic silicon carbide irradiated with heavy ion ( $\mathrm{Si}$ and $\mathrm{Fe}$ ) in an $\mathrm{MeV}$ energy range. ${ }^{14)}$ In the damaged but still crystalline region, very fine defect clusters with unclear images were observed at high density. The types and configurations of these clusters were not identified. The density of dislocations in the substrate, or the region beyond the ion range, associated with a plastic deformation was not high, considering the extent of swelling in the adjacent amorphized band. This observation supports that the plastic flow associated with the irradiationinduced swelling occurred mostly toward the constraint-free direction and consequently the degree of linear deformation in this experiment is corresponding to that of the isotropic deformation in unconstrained conditions.

\section{Conclusions}

An experimental technique to study the irradiation-induced volume changes and microstructural evolution in silicon carbide in accurately controlled irradiation conditions was successfully developed. The absolute accuracy in volume change measurement for the current technique is approximately $0.02 \%$, which is determined by the specimens' surface roughness.

Surface roughness of the irradiated silicon carbide appeared to increase approximately in proportion to the ion fluence. However, the irradiation-induced roughening was not significantly disturbing the measurement, since it was minor and seemed to be related with the degree of volume changes.

All the irradiated samples exhibited swelling that tended to saturate at certain fluences. The swelling increased with the increased ion fluence and the swelling rate and saturated volume decreased as temperature was elevated. The obtained temperature dependence of saturated swelling fell at the bottom of the reported neutron data band. A low fluence irradiation data suggested about $20 \%$ of surviving defect fraction to NRT-dpa at $333 \mathrm{~K}$. Variations in defect production rate caused only minor change in swelling rates and almost no effect on a saturated swelling value.

Amorphization was observed only at $333 \mathrm{~K}$. The threshold dose was determined to be $1.07 \mathrm{dpa}$ through an XTEM microstructural examination. The irradiated microstructure was high-density defect clusters with unclear images in TEM at $333 \mathrm{~K}$.

\section{Acknowledgements}

This work is supported by Laboratory for Complex Energy Processes, Institute of Advanced Energy, Kyoto University and Core Research for Evolutional Science and Technology (CREST), Japan Science and Technology Corporation (JST).

\section{REFERENCES}

1) A. Kohyama, Y. Katoh, T. Hinoki, W. Zhang and M. Kotani: Proceedings of the Eighth European Conference on Composite Materials, Vol. 4 (1998) 15-22. 


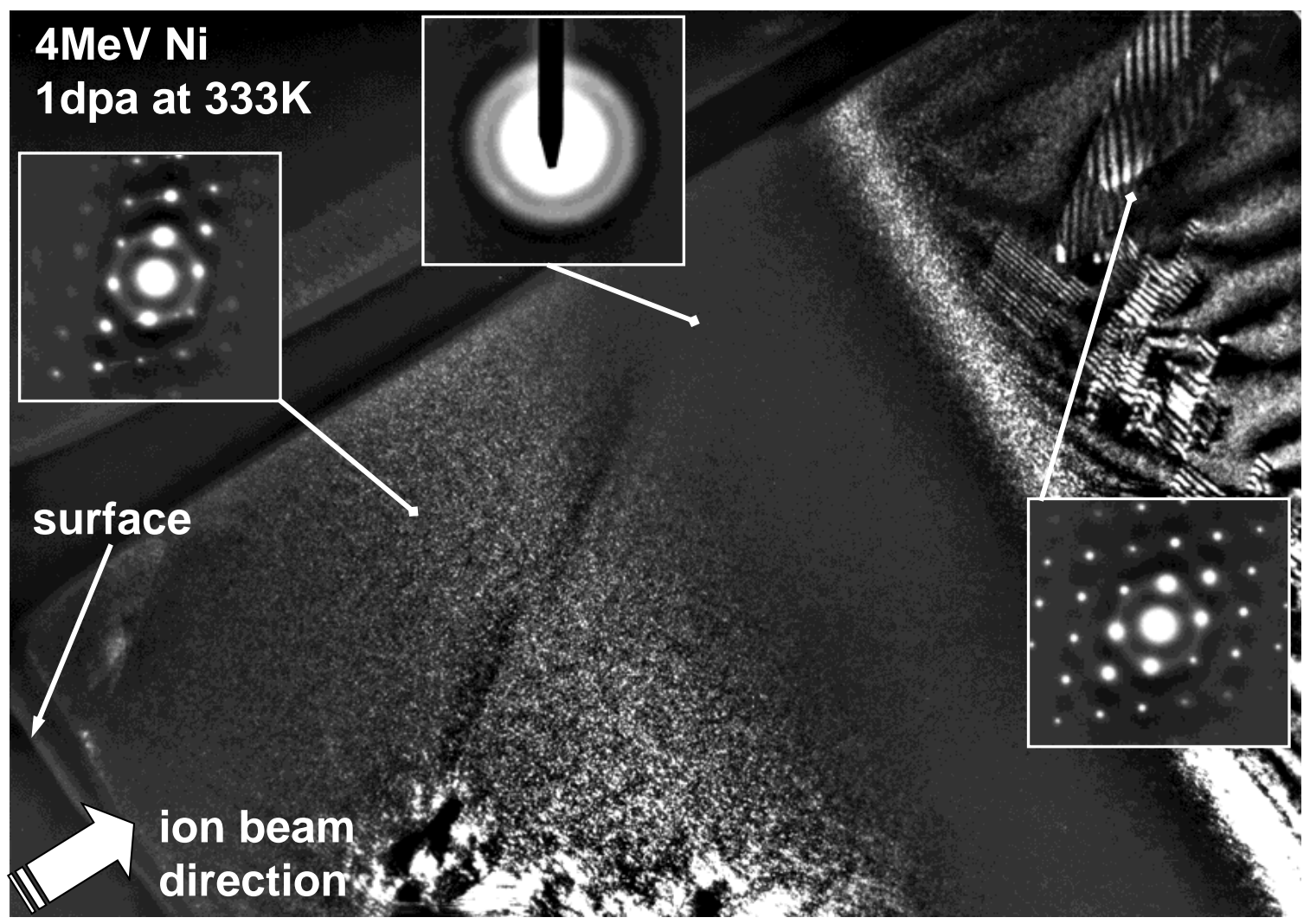

Fig. 7 Cross-sectional transmission electron micrograph of cubic silicon carbide irradiated with $4 \mathrm{MeV}$ nickel to $1 \mathrm{dpa}$ at $333 \mathrm{~K}$. $Z \sim\langle 110\rangle, g=\{111\}$.

2) P. Fenici, A. J. Frias Rebelo, R. H. Jones, A. Kohyama and L. L. Snead: J. Nucl. Mater. 258-263 (1998) 215-225.

3) S. J. Zinkle and C. Kinoshita: J. Nucl. Mater. 251 (1997) 200-217.

4) S. J. Zinkle and L. L. Snead: Fusion Materials, DOE/ER-0313/24 (1998) 93-114

5) A. Kohyama, Y. Katoh, M. Ando and K. Jimbo: Fusion Engineering and Design 51-52 (2000) 789-795.

6) J. F. Ziegler: Ion Beam Interactions with Matter, http://www.research.ibm.com/ionbeams/

7) H. L. Heinisch: Proc. of 2nd IEA/JUPITER Joint Int. Workshop on $\mathrm{SiC} / \mathrm{SiC}$ Ceramic Composites for Fusion Application (1997) 156-161.

8) Y. Katoh, A. Kohyama and T. Hinoki: Proceedings of the Eighth European Conference on Composite Materials, Vol. 4 (1998) 351-357.
9) B. N. Singh and S. J. Zinkle: J. Nucl. Mater. 206 (1993) 212-229.

10) K. Kitagawa, K. Yamakawa, H. Fukushima, T. Yoshiie, Y. Hayashi, H. Yoshida, Y. Shimomura and M. Kiritani: J. Nucl. Mater. 133\&134 (1985) 395-399.

11) C. J. McHargue and J. M. Williams: Nucl. Instrum. Meth. B80/81 (1993) 889-894.

12) H. Itoh, M. Yoshikawa, I. Nashiyama, H. Okamura, S. Misawa and S. Yoshida: J. Appl. Phys. 77 (1995) 837-842.

13) M. Kiritani: J. Nucl. Mater. 160 (1988) 135-141.

14) L. L. Snead and S. J. Zinkle: Mater. Res. Soc. Symp. Proc. 439 (1997) 595-606. 